\title{
ESTIMATES FOR THE ABSOLUTE RUIN PROBABILITY IN THE COMPOUND POISSON RISK MODEL WITH CREDIT AND DEBIT INTEREST
}

\author{
JINXIA ZHU,* \\ HAILIANG YANG, ${ }^{* *}$ The University of Hong Kong
}

\begin{abstract}
In this paper we consider a compound Poisson risk model where the insurer earns credit interest at a constant rate if the surplus is positive and pays out debit interest at another constant rate if the surplus is negative. Absolute ruin occurs at the moment when the surplus first drops below a critical value (a negative constant). We study the asymptotic properties of the absolute ruin probability of this model. First we investigate the asymptotic behavior of the absolute ruin probability when the claim size distribution is light tailed. Then we study the case where the common distribution of claim sizes are heavy tailed.
\end{abstract}

Keywords: Absolute ruin probability; credit and debit interest rates; compound Poisson model; heavy-tailed and light-tailed distributions; asymptotic results

2000 Mathematics Subject Classification: Primary 60K10; 60K20; 60F99

\section{Introduction}

We assume that the insurance company can invest its (positive) capital and earn interest with a constant force and that the company is still allowed to run its business when its surplus is negative but greater than the critical level by paying debit interest. The ruin defined accordingly is called the absolute ruin. Motivated by Cai (2007), we study the asymptotic behavior of the absolute ruin probabilities for both heavy-tailed and light-tailed claim size distributions. Our model can serve as a generalization of those ordinary ruin probabilities for risk models with constant interest force. Many of the results concerning the asymptotic properties of ruin probabilities are obtained under the constraint that the premium received per unit of time is greater than the average amount of claim per unit of time, which is called the positive safety loading condition in the case without investment. In this paper, however, we obtain results about the asymptotic behavior of the absolute ruin probabilities without such restriction.

Consider the continuous-time risk model, where claims arrive according to a homogeneous Poisson process with intensity rate $\lambda$ and premiums are collected continuously at a constant rate $p$. The amount of each claim is independent of each other and is also independent of the claim number process. Moreover, the insurance company earns credit interest with a constant force $r(r>0)$ when the surplus is positive and pays debit interest with a constant force of $\alpha$ when the surplus is negative. Let $S_{i}$ denote the arrival epoch of the $i$ th claim and $U_{i}$ its

Received 28 February 2008; revision received 27 June 2008.

* Current address: Australian School of Business, The University of New South Wales, Sydney, Australia.

Email address: jinxia.zhu@unsw.edu.au

** Postal address: Department of Statistics and Actuarial Science, The University of Hong Kong, Pokfulam Road, Hong Kong. Email address: hlyang@hkusua.hku.hk 
claim size. Then $N(t)=\sharp\left\{i: S_{i} \leq t\right\}$ denotes the number of claims occurring before and on time $t$ and it is a Poisson process with intensity rate $\lambda$. The sequence $\left\{U_{i}\right\}$ is identically and independently distributed with distribution function $F(\cdot)$ and it is independent of $N(t)$. We use $\mu_{F}$ to denote the expectation of the distribution $F$ and introduce the quantity $\rho=\lambda \mu_{F}$, the expected claim amount per unit time. When the surplus drops below 0 , the insurer could borrow money with the amount equal to the deficit at a debit interest force $\alpha>0$. It is reasonable to assume that $r \leq \alpha$. In the mean time, the insurer will repay the debts and the debt interest continuously. This leads to the following risk reserve process $\{R(t)\}$ with dynamics

$$
\mathrm{d} R(t)=(p+r R(t) \mathbf{1}\{R(t) \geq 0\}+\alpha R(t) \mathbf{1}\{R(t)<0\}) \mathrm{d} t-\mathrm{d} S(t),
$$

where $S(t)=\sum_{i=1}^{N(t)} U_{i}$ is the aggregate claim up to time $t$ and $\mathbf{1}\{\cdot\}$ is the indicator function.

Note from the above dynamics that the premium income will no longer be able to cover the debits when the surplus is less than $-p / \alpha$. That is, the surplus process will not be able to return to a positive amount whenever the process hits $-p / \alpha$ or any level below that. We call $-p / \alpha$ the critical value and, therefore, we define the time to absolute ruin as

$$
T=\inf \left\{t \geq 0: R(t) \leq-\frac{p}{\alpha}\right\} .
$$

We call this time the time of absolute ruin in the sense that the surplus will no longer be able to return to a positive level.

Suppose that $(\Omega, \mathcal{F}, \mathrm{P})$ is a probability space that carries all the subjects. Let $\left\{\mathcal{F}_{t}\right\}$ be the natural filtration generated by $\{R(t)\}$. It can be seen that $\{R(t), t\}$ is a Markov process and that $T$ is a stopping time. Let $\mathrm{P}_{x}(\cdot)=\mathrm{P}(\cdot \mid R(0)=x)$. Define the absolute ruin probability by

$$
\psi(u)=\mathrm{P}_{u}(T<\infty),
$$

and define the nonruin probability by

$$
\bar{\psi}(u)=\mathrm{P}_{u}(T=\infty) .
$$

Since, when the initial reserve is $-p / \alpha$, absolute ruin occurs immediately, we have

$$
\psi\left(-\frac{p}{\alpha}\right)=1 .
$$

Absolute ruin was first considered in Gerber (1971), (1979). Embrechts and Schmidli (1994) considered a general insurance risk model and used the piecewise-deterministic Markov process approach and the martingale methodology to study the absolute ruin probability. Dickson and dos Reis (1997) studied the absolute ruin problem for a compound Poisson model. They used simulation to estimate the probability of absolute ruin and to investigate the effect of interest on the time to recovery to surplus level 0 , the number of claims that occurred when the surplus was below 0 , and the maximum absolute value of the surplus process when it was below 0 . A risk model where the surplus process is modeled by a Brownian motion in the presence of investment and dividend payments according to a barrier strategy was included in Cai et al. (2006). Explicit expressions for the expectation of the discounted dividends are derived there. Cai (2007) studied the absolute ruin problem of the compound Poisson surplus process with only debit interest. Cai concluded that the absolute ruin probability is asymptotically in proportion to the classical ruin probability as the initial reserve goes to $\infty$. More generally, 
Gerber and Yang (2007) considered the compound Poisson risk model perturbed by diffusion with investment. They derived an integro-differential equation satisfied by the absolute ruin probability regarded as a function of the initial reserve and obtained closed-form solutions when there was only risk-free investment and the claim size distribution was exponential. Explicit expressions for the absolute ruin probability were also provided for the classical compound Poisson model with a constant interest rate.

For the asymptotic behavior of the absolute ruin probability, the presence of credit interest and debit interest complicates the problem. Explicit expressions for the absolute ruin probabilities have only been given in the case of an exponential claim size distribution. We are interested in estimating the absolute ruin probability for a given initial reserve $u$, in particular, when $u$ is large. The paper is organized as follows. In Section 2 we assume that the claim size distribution is heavy tailed, or more specifically, the equilibrium function of the claim size distribution is subexponential, and we show that the absolute ruin probability is also subexponentially decreasing for a large initial capital $u$. In Section 3 we consider the case where the claim size distribution has a light tail and we prove that the absolute ruin probability is exponentially decreasing.

\section{Equations for the probability of survival}

In the subsequent sections we always assume that the distribution function $F(x)$ is continuous. For convenience, we introduce the functions

$$
\psi_{+}(u)= \begin{cases}\psi(u) & \text { for } u \geq 0 \\ 0 & \text { for } u<0\end{cases}
$$

and

$$
\psi_{-}(u)= \begin{cases}0 & \text { for } u \geq 0 \\ \psi(u) & \text { for } u<0\end{cases}
$$

Obviously, absolute ruin can only occur at the moment a claim arrives. So absolute ruin cannot occur before the first claim. Therefore, we condition on the arrival time $S_{1}$ and the amount of the first claim, $U_{1}$. Write $\bar{s}_{t\urcorner r}=\int_{0}^{t} \mathrm{e}^{r s} \mathrm{~d} s$. Given that $S_{1}=t$ and $U_{1}=y$, the surplus just after the arrival of the first claim is $\left.R(0) \mathrm{e}^{r t}+p \bar{s}_{t}\right\urcorner r$ if $R(0) \geq 0$ and $\left.R(0)+p \bar{s}_{t}\right\urcorner \alpha$ if $-p / \alpha<R(0)<0$. Let

$$
\left.a_{+}(t)=u \mathrm{e}^{r t}+p \bar{s}_{t}\right\urcorner r \quad \text { for } u \geq 0, \quad t(u)=\frac{1}{\alpha} \log \frac{p}{\alpha u+p} \quad \text { for } u<0,
$$

and

$$
a_{-}(t)=u \mathrm{e}^{\alpha t}+p \bar{s}_{t\urcorner \alpha} \mathbf{1}\{t<t(u)\}+p \bar{s}_{t\urcorner r} \mathbf{1}\{t \geq t(u)\} \quad \text { for } u<0 .
$$

So the conditional probability that the company will not go bankrupt is $\bar{\psi}\left(a_{+}(t)\right)$ if $u \geq 0$ and $\bar{\psi}\left(a_{-}(t)\right)$ if $-p / \alpha<u<0$. Then we have

$$
\begin{aligned}
\overline{\psi_{+}}(u)=\int_{0}^{\infty} \lambda \mathrm{e}^{-\lambda t} \mathrm{~d} t & \left(\int_{0}^{a_{+}(t)} \psi_{+}\left(a_{+}(t)-y\right) \mathrm{d} F(y)\right. \\
& \left.+\int_{a_{+}(t)}^{a_{+}(t)+p / \alpha} \overline{\psi_{-}}\left(a_{+}(t)-y\right) \mathrm{d} F(y)\right) \quad \text { for } u \geq 0
\end{aligned}
$$


and

$$
\begin{aligned}
\overline{\psi_{-}}(u)= & \int_{0}^{t(u)} \lambda \mathrm{e}^{-\lambda t} \mathrm{~d} t \int_{0}^{a_{-}(t)+p / \alpha} \overline{\psi_{-}}\left(a_{-}(t)-y\right) \mathrm{d} F(y) \\
+ & \int_{t(u)}^{\infty} \lambda \mathrm{e}^{-\lambda t} \mathrm{~d} t\left(\int_{0}^{a_{-}(t)} \overline{\psi_{+}}\left(a_{-}(t)-y\right) \mathrm{d} F(y)\right. \\
& \left.+\int_{a_{-}(t)}^{a_{-}(t)+p / \alpha} \overline{\psi_{-}}\left(a_{-}(t)-y\right) \mathrm{d} F(y)\right) \text { for }-\frac{p}{\alpha}<u<0 .
\end{aligned}
$$

Changing variable according to $s=a_{-}(t)$ gives us

$$
\begin{aligned}
\overline{\psi_{+}}(u)= & \lambda(p+r u)^{\lambda / r} \int_{u}^{\infty}(p+r s)^{-\lambda / r-1} \mathrm{~d} s \\
& \times\left(\int_{0}^{s} \overline{\psi_{+}}(s-y) \mathrm{d} F(y)+\int_{s}^{s+p / \alpha} \overline{\psi_{-}}(s-y) \mathrm{d} F(y)\right) \quad \text { for } u \geq 0
\end{aligned}
$$

and

$$
\begin{aligned}
\overline{\psi_{-}}(u)= & \lambda(p+\alpha u)^{\lambda / \alpha} \int_{u}^{0}(p+\alpha s)^{-\lambda / \alpha-1} \mathrm{~d} s \int_{0}^{s+p / \alpha} \overline{\psi_{-}}(s-y) \mathrm{d} F(y) \\
+ & \lambda p^{\lambda / r} \mathrm{e}^{-\lambda t(u)} \int_{0}^{\infty}(p+r s)^{-\lambda / r-1} \mathrm{~d} s \\
& \times\left(\int_{0}^{s} \overline{\psi_{+}}(s-y) \mathrm{d} F(y)+\int_{s}^{s+p / \alpha} \overline{\psi_{-}}(s-y) \mathrm{d} F(y)\right) \quad \text { for }-\frac{p}{\alpha}<u<0 .
\end{aligned}
$$

Next, we will proceed to derive integro-differential equations for the probabilities of survival. Before doing this, we need to show that the functions concerned are differentiable. It has been shown for some continuous-time risk processes that the ruin probability as a function of the initial reserve is smooth if the claim size distribution function is regular enough. These kind of analytic properties for the ruin probability or some other frequently concerned ruin functions with respect to the initial capital $u$ have been considered in the literature for some other models. Zhu and Yang (2006) obtained sufficient conditions on the claim size distributions for the continuity and differentiability (of higher order). Without much modification, the technique used there can be adopted to show that the absolute ruin probability in this model is differentiable if the claim size distribution function $F(x)$ is continuous.

By differentiating (2.1) and (2.2) with respect to $u$, we obtain

$$
\begin{aligned}
{\overline{\psi_{+}}}^{\prime}(u)= & \frac{\lambda}{p+r u} \overline{\psi_{+}}(u)-\frac{\lambda}{p+r u} \int_{0}^{u} \overline{\psi_{+}}(u-y) \mathrm{d} F(y) \\
& -\frac{\lambda}{p+r u} \int_{u}^{u+p / r} \overline{\psi_{-}}(u-y) \mathrm{d} F(y) \quad \text { for } u>0, \\
{\overline{\psi_{-}}}^{\prime}(u)= & \frac{\lambda}{p+\alpha u} \overline{\psi_{-}}(u)-\frac{\lambda}{p+\alpha u} \int_{0}^{u+p / \alpha} \overline{\psi_{-}}(u-y) \mathrm{d} F(y) \quad \text { for }-\frac{p}{\alpha}<u<0 .
\end{aligned}
$$

We point out here that the integro-differential equation for $\overline{\psi_{-}}(u),(2.4)$, is independent of $\overline{\psi_{+}}(u)$. But the solution $\overline{\psi_{-}}(u)$ is subject to certain boundary conditions which may involve $\overline{\psi_{+}}(u)$. 
Note that (2.3) can be rewritten as

$$
(p+r u) \overline{\psi_{+}}(u)=\lambda \overline{\psi_{+}}(u)-\lambda \int_{0}^{u} \overline{\psi_{+}}(u-y) \mathrm{d} F(y)-\lambda \int_{u}^{u+p / \alpha} \overline{\psi_{-}}(u-y) \mathrm{d} F(y) .
$$

Define

$$
g(u)=\int_{0}^{u} \mathrm{~d} x \int_{x}^{x+p / \alpha} \overline{\psi_{-}}(u-y) \mathrm{d} F(y) .
$$

Replacing $u$ by $x$ in the integro-differential equation (2.5) and integrating both sides from 0 to $u$ yields, for $u>0$,

$$
(p+r u) \overline{\psi_{+}}(u)-p \overline{\psi_{+}}(0)-r \int_{0}^{u} \overline{\psi_{+}}(x) \mathrm{d} x=\lambda \int_{0}^{u} \overline{\psi_{+}}(u-y) \bar{F}(y) \mathrm{d} y-\lambda g(u),
$$

where $\bar{F}(y)=1-F(y)$, i.e.

$$
\begin{aligned}
\overline{\psi_{+}}(u)= & \frac{p}{p+r u} \overline{\psi_{+}}(0)+\frac{r}{p+r u} \int_{0}^{u} \overline{\psi_{+}}(u-y) \mathrm{d} y \\
& +\frac{\lambda}{p+r u} \int_{0}^{u} \overline{\psi_{+}}(u-y) \bar{F}(y) \mathrm{d} y-\frac{\lambda}{p+r u} g(u) .
\end{aligned}
$$

For convenience, we introduce the function

$$
G(u)=\frac{\overline{\psi_{+}}(u)-\overline{\psi_{+}}(0)}{1-\overline{\psi_{+}}(0)} \quad \text { for } u \geq 0,
$$

and study this function instead. Then, $\overline{\psi_{+}}(u)=\overline{\psi_{+}}(0)+\psi_{+}(0) G(u)$. Substituting this into (2.7), we have

$$
\begin{aligned}
(p+r u) G(u)= & \lambda \frac{\overline{\psi_{+}}(0)}{\psi_{+}(0)} \int_{0}^{u} \bar{F}(y) \mathrm{d} y+\lambda \int_{0}^{u} G(u-y) \bar{F}(y) \mathrm{d} y \\
& +r \int_{0}^{u} G(y) \mathrm{d} y-\lambda \frac{1}{\psi_{+}(0)} g(u) .
\end{aligned}
$$

Let $\rho=\lambda \mu_{F}, b=\lambda / \psi_{+}(0)$, and $K=\rho \overline{\psi_{+}}(0) / \psi_{+}(0)$. Let $F_{\mathrm{e}}(u)$ be the equilibrium distribution function associated with the distribution function $F(u)$, i.e.

$$
F_{\mathrm{e}}(u)=\mu_{F}^{-1} \int_{0}^{u} \bar{F}(x) \mathrm{d} x .
$$

Then, (2.8) can be rewritten as

$$
p G(u)+r u G(u)=r \int_{0}^{u} G(y) \mathrm{d} y+K F_{\mathrm{e}}(u)+\rho G * F_{\mathrm{e}}(u)-b g(u),
$$

where ' $*$ ' stands for the Stieltjes convolution. 


\section{Heavy-tailed claim size}

In this section we consider the case where the claim size distribution is heavy tailed and obtain an asymptotic formula for the absolute ruin probability. First, we introduce a special class of heavy-tailed distributions and some of their properties.

Definition 3.1. A distribution function $F(x)$ with support $(0, \infty)$ is subexponential if

$$
\lim _{x \rightarrow \infty} \frac{\overline{F^{* n}}(x)}{\bar{F}(x)}=n \text { for some } n \geq 2 .
$$

Use $\&$ to denote the class of subexponential distribution functions. An alternative definition of the subexponetial distribution function is stated below.

Definition 3.2. Let $\left(X_{i}\right)_{i \in \mathbb{N}}$ be independent and identically distributed random variables with distribution function $F(x)$ with support $(0, \infty)$. Then $F(x)$ is a subexponential distribution function if

$$
\lim _{x \rightarrow \infty} \frac{\mathrm{P}\left(X_{1}+\cdots+X_{n}>x\right)}{\mathrm{P}\left(\max \left(X_{1}, \ldots, X_{n}\right)>x\right)}=1 \quad \text { for some } n \geq 2 .
$$

Definition 3.2 demonstrates the heavy tailedness of subexponential distribution functions.

Lemma 3.1. If $F \in s$ then

$$
\begin{gathered}
\lim _{x \rightarrow \infty} \frac{\bar{F}(x-y)}{\bar{F}(x)}=1 \quad \text { for any } y \in \mathbb{R}, \\
\int_{0}^{\infty} \mathrm{e}^{\varepsilon x} \mathrm{~d} F(x)=\infty \text { for any } \varepsilon>0 \\
\frac{\bar{F}(x)}{\mathrm{e}^{-\varepsilon x}} \rightarrow \infty \text { as } x \rightarrow \infty \text { for any } \varepsilon>0 .
\end{gathered}
$$

The name subexponential distribution function arises from property (3.3): the tail of the distribution function decreases more slowly than any exponential tail. Property (3.2) shows that subexponential distribution functions have no exponential moments. In the following we use the notation ' $\sim$ ' to denote the asymptotic equivalence between two functions of the same variable as this variable goes to $\infty$.

Lemma 3.2. (i) If the distributions $F \in \&$ and $\overline{G_{i}}(x) \sim c_{i} \bar{F}(x)$, where $c_{i} \in(0, \infty)$ for $i=1,2$, then

$$
\overline{G_{1} * G_{2}}(x) \sim\left(c_{1}+c_{2}\right) \bar{F}(x) .
$$

(ii) If the distributions $F \in \&$ and $\bar{G}(x) \sim c \bar{F}(x)$, where $c \in[0, \infty)$, then

$$
\overline{F * G}(x) \sim(1+c) \bar{F}(x) .
$$

Lemma 3.3. Suppose that $p_{n} \geq 0$ for all $n=0,1, \ldots, \sum_{n=0}^{\infty} p_{n}=1$, and $\sum_{n=0}^{\infty} p_{n}(1+\varepsilon)^{n}<$ $\infty$ for some $\varepsilon>0$, and let the distribution $F \in$ \&. Then

$$
1-\sum_{n=0}^{\infty} p_{n} F^{n *}(x) \sim \sum_{n=0}^{\infty} n p_{n} \bar{F}(x) .
$$


Subexponential distributions can be used to model claims with extremely large values, which is often seen in insurance, known as catastrophe insurance. For more information about this class of distributions, we refer the reader to Embrechts et al. (1997).

Next we will assume that the integrated tail distribution function $F_{\mathrm{e}}(x)$ is subexponential. We are interested in the asymptotic behavior of the absolute ruin probability for large $u$. Cai (2007) studied the same problem when the credit interest was 0 , but the technique used there does not apply to our case where positive capital earns interest. The approach we use here is motivated by Kalashnikov and Konstantinides (2000).

Introduce an auxiliary function

$$
k(u)=\int_{u}^{\infty} y \mathrm{~d} G(y) \quad \text { for } u \geq 0 .
$$

Lemma 3.4. Assume that $F(x)$ is continuous, $p>\lambda \mu_{F}$, and that $F_{\mathrm{e}}(u) \in 8$. Then

$$
k(u) \sim \frac{\rho+K}{r}\left(1-F_{\mathrm{e}}(u)\right) .
$$

Proof. Noting that $\int_{0}^{u} G(y) \mathrm{d} y=u G(u)-\int_{0}^{u} y \mathrm{~d} G(y)$, it follows from (2.9) that

$$
p G(u)=-r \int_{0}^{u} y \mathrm{~d} G(y)+K F_{\mathrm{e}}(u)-b g(u)+\rho G * F_{\mathrm{e}}(u),
$$

i.e.

$$
G(u)=-\frac{r}{p}(k(0)-k(u))+\frac{K}{p} F_{\mathrm{e}}(u)-\frac{b}{p} g(u)+m G * F_{\mathrm{e}}(u),
$$

where $m=\rho / p<1$. Letting $u$ converge to $\infty$, we have

$$
1=-\frac{r}{p} k(0)+\frac{K}{p}-\frac{b}{p} g(\infty)+m .
$$

Define

$$
H(u)=\sum_{n=0}^{\infty} m^{n} F_{\mathrm{e}}^{* n}(u)
$$

and

$$
\eta(u)=k(0)-k(u) .
$$

Note that $F_{\mathrm{e}} * H(u)=(H(u)-1) / m$. Then from (3.4) we have

$$
G(u)=-\frac{r}{p} \eta * H(u)+\frac{K}{p} F_{\mathrm{e}} * H(u)-\frac{b}{p} g * H(u) .
$$

Applying the Laplace-Stieltjes transform to (3.7) gives

$$
\hat{G}(s)=-\frac{r}{p} \hat{\eta}(s) \hat{H}(s)+\frac{K}{p} \hat{F}_{\mathrm{e}}(s) \hat{H}(s)-\frac{b}{p} \hat{g}(s) \hat{H}(s),
$$

where $\hat{F}_{\mathrm{e}}(\cdot)$ and $\hat{g}(\cdot)$ are the Laplace-Stieltjes transform of $F_{\mathrm{e}}(\cdot)$ and $g(\cdot)$, respectively. Noting that

$$
\hat{H}(s)=\frac{1}{1-m \hat{F}_{\mathrm{e}}(s)},
$$


it follows from (3.8) that

$$
\hat{\eta}(s)=-\frac{p}{r} \hat{G}(s)+\frac{\rho}{r} \hat{G}(s) \hat{F}_{\mathrm{e}}(s)+\frac{K}{r} \hat{F}_{\mathrm{e}}(s)-\frac{b}{r} \hat{g}(s),
$$

where $\hat{G}(\cdot)$ is the Laplace-Stieltjes transform of $G(\cdot)$. Inverting the above relation yields

$$
\eta(u)=-\frac{p}{r} G(u)+\frac{\rho}{r} G * F_{\mathrm{e}}(u)+\frac{K}{r} F_{\mathrm{e}}(u)-\frac{b}{r} g(u) .
$$

By (3.5) we obtain

$$
\begin{aligned}
k(u)= & k(0)-\eta(u) \\
= & -\frac{p}{r}(1-G(u))+\frac{\rho}{r}(1-G) * F_{\mathrm{e}}(u)+\frac{K+\rho}{r}\left(1-F_{\mathrm{e}}(u)\right) \\
& -\frac{b}{r}(g(\infty)-g(u)) .
\end{aligned}
$$

By definition (2.6) we see that

$$
\begin{aligned}
g(\infty)-g(u) & =\int_{u}^{\infty} \mathrm{d} x \int_{x}^{x+p / \alpha} \overline{\psi_{-}}(u-y) \mathrm{d} F(y) \\
& \leq \mu_{F}\left(\overline{F_{\mathrm{e}}}(u)-\overline{F_{\mathrm{e}}}\left(u+\frac{p}{\alpha}\right)\right) \\
& \sim o\left(\overline{F_{\mathrm{e}}}(u)\right),
\end{aligned}
$$

where the last equivalence follows from (3.1). Noting that

$$
k(u) \geq u(1-G(u)),
$$

it follows from (3.9) that

$$
k(u) \geq\left(1+\frac{p}{r u}\right)^{-1}\left(\frac{K+\rho}{r}\left(1-F_{\mathrm{e}}(u)\right)-\frac{b}{r}(g(\infty)-g(u))\right) .
$$

Since $\eta * H(u)=\int_{0}^{u} \eta(u-y) \mathrm{d} H(y) \leq \eta(u) H(u)$, from (3.7), it follows that

$$
G(u) \geq-\frac{r}{p} \eta(u) H(u)+\frac{K}{p} F_{\mathrm{e}} * H(u)-\frac{b}{p} g * H(u) .
$$

Hence,

$$
\eta(u) \geq \frac{K F_{\mathrm{e}} * H(u)-b g * H(u)-p G(u)}{r H(u)} .
$$

As a result,

$$
k(u) \leq k(0)-\frac{K F_{\mathrm{e}} * H(u)-b g * H(u)-p G(u)}{r H(u)} .
$$

For any bounded positive increasing function $f$ with support $(0, \infty)$, let $\tilde{f}(x)$ denote the normalized function $f(x) / f(\infty)$. Then, by (3.5) and (3.12), we obtain

$$
\begin{gathered}
k(u) \leq r(H(u))^{-1}\left(-r k(0) H(\infty)(1-\tilde{H}(u))+K H(\infty)\left(1-F_{\mathrm{e}} * \tilde{H}(u)\right)\right. \\
-b g(\infty) H(\infty)(1-\tilde{g} * \tilde{H}(u))) .
\end{gathered}
$$


Note that, by Lemma 3.3 and (3.6), we have

$$
1-\tilde{H}(u) \sim \sum_{n=0}^{\infty}(1-m) n m^{n}\left(1-F_{\mathrm{e}}(u)\right)=\frac{m}{1-m}\left(1-F_{\mathrm{e}}(u)\right)
$$

and by (3.10) we have

$$
\tilde{g}(u) \sim o\left(1-F_{\mathrm{e}}(u)\right) .
$$

As a result, by Lemma 3.2 we obtain

$$
\begin{aligned}
1-F_{\mathrm{e}} * \tilde{H}(x) & \sim \frac{1}{1-m} \overline{F_{\mathrm{e}}}(x), \\
1-\tilde{g} * \tilde{H}(x) & \sim \frac{m}{1-m} \overline{F_{\mathrm{e}}}(x) .
\end{aligned}
$$

Then, it follows that the right-hand side of (3.13) is asymptotically equivalent to $((\rho+K) / r)(1-$ $\left.F_{\mathrm{e}}(u)\right)$. By Lemma 3.2 again, (3.5), and (3.10), we see that the right-hand side of (3.11) is also asymptotically equivalent to $((\rho+K) / r)\left(1-F_{\mathrm{e}}(u)\right)$. This concludes the proof.

Theorem 3.1. If $F$ is continuous, the equilibrium distribution function $F_{\mathrm{e}}(u)$ is subexponential, and

$$
\limsup _{u \rightarrow \infty}\left(\frac{\overline{F_{\mathrm{e}}}(u)}{u}\right)^{-1} \int_{u}^{\infty} \frac{\overline{F_{\mathrm{e}}}(y)}{y^{2}} \mathrm{~d} y<1
$$

then

$$
\psi(u) \sim \frac{\lambda}{r} \int_{u}^{\infty} \frac{\bar{F}(y)}{y} \mathrm{~d} y .
$$

Proof. First, we assume that $p>\lambda \mu_{F}$. From the definition of $k(u)$ and noting that $\lim _{u \rightarrow \infty} k(u) / u=0$, we have

$$
1-G(u)=-\int_{u}^{\infty} \frac{1}{y} \mathrm{~d} k(y)=\frac{k(u)}{u}-\int_{u}^{\infty} \frac{k(y)}{y^{2}} \mathrm{~d} y .
$$

By Lemma 3.4, it follows that

$$
\begin{aligned}
\frac{k(u)}{u} & \sim \frac{\rho+K}{r} \frac{1-F_{\mathrm{e}}(u)}{u}, \\
\int_{u}^{\infty} \frac{k(y)}{y^{2}} \mathrm{~d} y & \sim \frac{\rho+K}{r} \int_{u}^{\infty} \frac{1-F_{\mathrm{e}}(y)}{y^{2}} \mathrm{~d} y .
\end{aligned}
$$

Note that, for any positive functions $a_{1}(x), a_{2}(x), b_{1}(x)$, and $b_{2}(x)$, with $a_{1}(x) \sim b_{1}(x)$, $a_{2}(x) \sim b_{2}(x)$, and $b_{1}(x) \geq b_{2}(x)$, there exists, for any $\varepsilon>0$, an $M>0$ such that, for any $x \geq M$,

$$
\left|a_{1}(x)-b_{1}(x)\right|<b_{1}(x) \varepsilon
$$

and

$$
\left|a_{2}(x)-b_{2}(x)\right|<b_{2}(x) \varepsilon .
$$


Therefore,

$$
\begin{aligned}
\left|\frac{a_{1}(x)-a_{2}(x)}{b_{1}(x)-b_{2}(x)}-1\right| & =\left|\frac{a_{1}(x)-b_{1}(x)-\left(a_{2}(x)-b_{2}(x)\right)}{b_{1}(x)-b_{2}(x)}\right| \\
& \leq \frac{b_{1}(x) \varepsilon+b_{2}(x) \varepsilon}{\left|b_{1}(x)-b_{2}(x)\right|} \\
& =\frac{b_{1}(x)+b_{2}(x)}{b_{1}(x)-b_{2}(x)} \varepsilon \\
& =\frac{1+b_{2}(x) / b_{1}(x)}{1-b_{2}(x) / b_{1}(x)} \varepsilon .
\end{aligned}
$$

If we have $\lim \sup _{x \rightarrow \infty} b_{2}(x) / b_{1}(x)<1$ then it follows that

$$
a_{1}(x)-a_{2}(x) \sim b_{1}(x)-b_{2}(x) .
$$

Hence, by (2.2)-(2.5), we obtain

$$
\begin{aligned}
1-G(u) & \sim \frac{\rho+K}{r}\left(\frac{1-F_{\mathrm{e}}(u)}{u}-\int_{u}^{\infty} \frac{1-F_{\mathrm{e}}(y)}{y^{2}} \mathrm{~d} y\right) \\
& =\frac{\rho+K}{r} \int_{u}^{\infty} \frac{\mathrm{d} \overline{F_{\mathrm{e}}}(y)}{y} .
\end{aligned}
$$

Consequently,

$$
\psi_{+}(u)=\psi_{+}(0)(1-G(u)) \sim \frac{\lambda}{r} \int_{u}^{\infty} \frac{\bar{F}(y)}{y} \mathrm{~d} y .
$$

Next, we consider the case in which $\lambda \mu_{F} \geq p$. Let $S_{u, p}(t)$ denote the surplus at time $t$ of a risk process following the dynamics (1.1) and with premium rate $p$ and initial value $u$. Then, for any $u>0$ and $0 \leq u_{0} \leq u, S_{u-u_{0}, p+r u_{0}}(t)=S_{u, p}(t)-u_{0}$ when $S_{u-u_{0}, p+r u_{0}}(t) \geq 0$ or, equivalently, $S_{u, p}(t) \geq u_{0}$. After the time that $S_{u, p}(t)$ first falls below $u_{0}, S_{u-u_{0}, p+r u_{0}}(t) \leq$ $S_{u, p}(t)-u_{0}$. Note that the critical level for the process $S_{u, p}(t)$ is $-p / \alpha$, while the critical level for $S_{u-u_{0}, p+r u_{0}}(t)$ is $-p / \alpha-(r / \alpha) u_{0} \geq-p / \alpha-u_{0}$. Therefore, if we let $\phi(u, p)$ denote the ruin probability corresponding to the process $S_{u, p}(t)$, we can see that

$$
\phi(u, p) \leq \phi\left(u-u_{0}, p+r u_{0}\right) .
$$

Choose $u_{0}=\left(\lambda \mu_{F}-p+\varepsilon\right) / r$ for some small $\varepsilon>0$. Then, $p+r u_{0}>\lambda \mu_{F}$. Hence, by (3.15),

$$
\phi\left(u-u_{0}, p+r u_{0}\right) \sim \frac{\lambda}{r} \int_{u-u_{0}}^{\infty} \frac{\bar{F}(y)}{y} \mathrm{~d} y \sim \frac{\lambda}{r} \int_{u}^{\infty} \frac{\bar{F}(y)}{y} \mathrm{~d} y .
$$

Since $p \leq \lambda \mu_{F}$, it is easy to obtain, for any $\varepsilon>0$

$$
\phi(u, p) \geq \phi\left(u, \lambda \mu_{F}+\varepsilon\right) \sim \frac{\lambda}{r} \int_{u}^{\infty} \frac{\bar{F}(y)}{y} \mathrm{~d} y,
$$

where the last equivalence follows from (3.15) by noting that the premium rate $\lambda \mu_{F}+\varepsilon$ is strictly greater than $\lambda \mu_{F}$. Combining (3.16), (3.17), and (3.18), we can conclude that (3.14) also holds for $\lambda \mu_{F} \geq p$.

Remark 3.1. Letting $\alpha \rightarrow \infty$, the model reduces to the ordinary ruin case. Here, our asymptotic result holds without restricting $p>\lambda \mu_{F}$. 


\section{Light-tailed claim size}

We consider the case that the claim size distribution is light tailed. Use $\hat{f}(s)$ or $L(f(u), u, s)$ to denote the Laplace-Stieltjes transform of the function $f(u)$ with respect to $u$ with dummy parameter $s$. Namely,

$$
\hat{f}(s)=L(f(u), u, s)=\int_{0}^{\infty} \mathrm{e}^{-s u} \mathrm{~d} f(u)
$$

Note that $L(u G(u), u, s)=L\left(\int_{0}^{u} G(x) \mathrm{d} x, u, s\right)-\hat{G}^{\prime}(s)$. Applying the Laplace-Stieltjes transform to both sides of (2.9), we find that

$$
p \hat{G}(u)-r \hat{G}^{\prime}(u)=K \hat{F}_{\mathrm{e}}(u)+\rho \hat{G}(s) \hat{F}_{\mathrm{e}}(s)-b \hat{g}(s) .
$$

Introduce

$$
\chi(x)=\int_{0}^{x}\left(p-\rho \hat{F}_{\mathrm{e}}(y)\right) \mathrm{d} y .
$$

Since $\chi(x) \rightarrow \infty$ as $x \rightarrow \infty$, solving the ordinary differential equation (4.1) gives

$$
\hat{G}(s)=\frac{1}{r} \int_{s}^{\infty} \mathrm{e}^{-(\chi(v)-\chi(s)) / r}\left(K \hat{F}_{\mathrm{e}}(v)-b \hat{g}(v)\right) \mathrm{d} v .
$$

Relation (4.2) can be used to obtain asymptotic results about the ruin probabilities in the lighttailed case.

Theorem 4.1. If $F(x)$ is a continuous distribution function and the equilibrium distribution $F_{\mathrm{e}}(x)$ is exponentially bounded, i.e. $1-F_{\mathrm{e}}(x) \leq C \mathrm{e}^{-a x}$ for some positive constants a and $C$, then, for any $\varepsilon>0$,

$$
\begin{gathered}
\psi(u)=o\left(\mathrm{e}^{-(a-\varepsilon) u}\right), \\
\psi(u) \mathrm{e}^{(a+\varepsilon) u} \rightarrow \infty \quad \text { as } u \rightarrow \infty .
\end{gathered}
$$

Proof. We can obviously see that $-a$ is the negative abscissa of convergence of $\hat{F}_{\mathrm{e}}(s)$. Noting that $\left(1-\hat{F}_{\mathrm{e}}(s)\right) / s=\int_{0}^{\infty} \mathrm{e}^{-s x} \overline{F_{\mathrm{e}}}(x) \mathrm{d} x$, we have $\hat{F}_{\mathrm{e}}(s) \leq 1$ if $s \geq 0$ and $\hat{F}_{\mathrm{e}}(s) \leq$ $\mathrm{Ca} /(s+a)+1-C$ if $-a<s<0$. Next, we show that

$$
\lim _{v \rightarrow \infty} \frac{\int_{0}^{v} \hat{F}_{\mathrm{e}}(x) \mathrm{d} x}{v}=0 .
$$

This is obviously true if $\int_{0}^{\infty} \hat{F}_{\mathrm{e}}(x) \mathrm{d} x<\infty$. If $\int_{0}^{\infty} \hat{F}_{\mathrm{e}}(x) \mathrm{d} x=\infty$, by L'Hospital's law we can show that (4.3) is still true since $\lim _{s \rightarrow \infty} \hat{F}_{\mathrm{e}}(s)=0$. It readily follows from (4.3) that, for any $s>-a$ and any $0<\varepsilon_{0}<p / r$,

$$
\exp \left(-\frac{p}{r}(v-s)+\frac{\rho}{r} \int_{s}^{v} \hat{F}_{\mathrm{e}}(x) \mathrm{d} x\right)=o\left(\exp \left(-\varepsilon_{0} v\right)\right) \quad \text { as } v \rightarrow \infty .
$$

Hence, we can see that, for any $s>-a$,

$$
\int_{s}^{\infty} \exp \left(-\frac{p}{r}(v-s)+\frac{\rho}{r} \int_{s}^{v} \hat{F}_{\mathrm{e}}(x) \mathrm{d} x\right) \mathrm{d} v<\infty .
$$


Using integration by parts, we obtain, for $s>-a$,

$$
\begin{aligned}
\int_{s}^{\infty} & \mathrm{e}^{-(\chi(v)-\chi(s)) / r} \hat{F}_{\mathrm{e}}(v) \mathrm{d} v \\
& =\int_{s}^{\infty} \exp \left(-\frac{p}{r}(v-s)+\frac{\rho}{r} \int_{s}^{v} \hat{F}_{\mathrm{e}}(x) \mathrm{d} x\right) \hat{F}_{\mathrm{e}}(v) \mathrm{d} v \\
& =\frac{r}{\rho} \int_{s}^{\infty} \exp \left(-\frac{p}{r}(v-s)\right) \mathrm{d} \exp \left(\frac{\rho}{r} \int_{s}^{v} \hat{F}_{\mathrm{e}}(x) \mathrm{d} x\right) \\
& =-\frac{r}{\rho}+\frac{p}{\rho} \int_{s}^{\infty} \exp \left(-\frac{p}{r}(v-s)+\frac{\rho}{r} \int_{s}^{v} \hat{F}_{\mathrm{e}}(x) \mathrm{d} x\right) \mathrm{d} v
\end{aligned}
$$

It easily follows, from (4.4) and (4.5), that the function $\int_{s}^{\infty} \mathrm{e}^{-(\chi(v)-\chi(s)) / r} \hat{F}_{\mathrm{e}}(v) \mathrm{d} v$ is convergent for all $s>-a$.

For all $s<-a$, as $\hat{F}_{\mathrm{e}}(s)=\infty$, we can conclude that the function $\int_{s}^{\infty} \mathrm{e}^{-(\chi(v)-\chi(s)) / r} \hat{F}_{\mathrm{e}}(v) \mathrm{d} v$ is not convergent. Consequently, we have

$$
\int_{s}^{\infty} \mathrm{e}^{-(\chi(v)-\chi(s)) / r} \hat{F}_{\mathrm{e}}(v) \mathrm{d} v \begin{cases}<\infty & \text { for }-a<s<\infty \\ =\infty & \text { for } s<-a\end{cases}
$$

Since it follows from (2.6) that

$$
\hat{g}(v) \leq \int_{0}^{\infty} \mathrm{e}^{-v x} \mathrm{~d} x \int_{x}^{x+p / \alpha} \mathrm{d} F(y) \leq \int_{0}^{\infty} \mathrm{e}^{-v x} \bar{F}(x) \mathrm{d} x=\mu_{F} \hat{F}_{\mathrm{e}}(v),
$$

we can see that

$$
\int_{s}^{\infty} \mathrm{e}^{-(\chi(v)-\chi(s)) / r} \hat{g}(v) \mathrm{d} v \leq \mu_{F} \int_{s}^{\infty} \mathrm{e}^{-(\chi(v)-\chi(s)) / r} \hat{F}_{\mathrm{e}}(v) \mathrm{d} v .
$$

Then it follows, from (4.2), (4.6), and (4.7), that $-a$ is the abscissa of the convergence of the Laplace-Stieltjes transform $\hat{G}(s)$. By Corollary 2.1 and Theorem 2.2b of Widder (1946), we deduce the asserted result.

Remark 4.1. Letting $\alpha \rightarrow \infty$, the absolute ruin problem reduces to the ordinary ruin problem.

In Theorem 4.1 we derive the same result as in Theorem 2 of Embrechts and Schmidli (1994). In that paper the authors made a lot effort in constructing a positive martingale and using the martingale convergence theorem to express the ruin probability in terms of a certain function. The result there holds under the assumption that $p>\lambda \mu_{F}$. Here we demonstrate another way to prove the asymptotic result when $p>\lambda \mu_{F}$ and, more importantly, Theorem 4.1 extends the result to the case where $p \leq \lambda \mu_{F}$.

\section{Acknowledgements}

We would like to thank the anonymous referee for helpful comments and suggestions. This research was supported by the Research Grants Council of the Hong Kong Special Administrative Region, China (project number 7050/05P). 


\section{References}

CAI, J. (2007). On the time value of absolute ruin with debit interest. Adv. Appl. Prob. 39, 343-359.

CaI, J., Gerber, H. U. and Yang, H. (2006). Optimal dividends in an Ornstein-Uhlenbeck type model. N. Amer. Actuarial J. 10, 94-119.

Dickson, D. C. AND dos ReIs, A. D. E. (1997). The effect of interest on negative surplus. Insurance Math. Econom. 21, 1-16.

Embrechts, P. ANd Schmidli, H. (1994). Ruin estimation for a general insurance risk model. Adv. Appl. Prob. 26, 404-422.

Embrechts, P., Klüppelberg, C. AND Mikosch, T. (1997). Modelling Extremal Events for Insurance and Finance. Springer, Berlin.

Gerber, H. U. (1971). Der Einfluss von Zins auf die Ruinwahrscheinlichkeit. Bull. Swiss Assoc. Actuaries 71, 63-70.

Gerber, H. U. (1979). An Introduction to Mathematical Risk Theory. S. S. Huebner Foundation, University of Pennsylvania, Philadelphia.

Gerber, H. U. ANd Yang, H. (2007). Absolute ruin probabilities in a jump diffusion risk model with investment. N. Amer. Actuarial J. 11, 159-169.

Kalashnikov, V. and Konstantinides, D. (2000). Ruin under interest force and subexponential claims: a simple treatment. Insurance Math. Econom. 27, 145-159.

WidDER, D. (1946). The Laplace Transform. Princeton University Press.

ZHU, J. AND YANG, H. (2006). On differentiability of ruin functions under Markov-modulated models. Submitted. 